\title{
Atom interferometry using wave packets with constant spatial displacements
}

\author{
Edward J. Su, ${ }^{1, *}$ Saijun Wu, ${ }^{1,2, \dagger}$ and Mara G. Prentiss ${ }^{1}$ \\ ${ }^{1}$ Harvard University Department of Physics, Cambridge, Massachusetts 02138, USA \\ ${ }^{2}$ Harvard University School of Engineering and Applied Science, Cambridge, Massachusetts 02138, USA
}

(Received 6 February 2010; published 27 April 2010)

\begin{abstract}
A standing-wave light-pulse sequence is demonstrated that places atoms into a superposition of wave packets with precisely controlled displacements that remain constant for times as long as $1 \mathrm{~s}$. The separated wave packets are subsequently recombined, resulting in atom interference patterns that probe energy differences of $\approx 10^{-34} \mathrm{~J}$ and can provide acceleration measurements that are insensitive to platform vibrations.
\end{abstract}

DOI: 10.1103/PhysRevA.81.043631

PACS number(s): 37.25.+k, 03.75.Dg

\section{INTRODUCTION}

Atom interferometry employs the interference of atomic de Broglie waves for precision measurements [1]. In practice, two effects limit the ultimate sensitivity of devices where the interfering atomic wave packets are allowed to propagate in free space: the effect of external gravitational fields upon the atomic trajectories, and transverse expansion of the atom cloud. By accounting for gravity, atomic fountains can increase the interrogation time during which the interferometry phase shifts accumulate [2]; alternatively one can use magnetic dipole forces to balance the force of gravity [3]. Magnetic waveguides $[4,5]$ can trap atoms for times longer than a second, suggesting the possibility of measuring energy differences between interfering wave packets with an uncertainty $<\hbar /(1 \mathrm{~s})$ $\sim 10^{-34} \mathrm{~J}$; however, this remarkable precision cannot be obtained if the decoherence time of the atoms is much shorter than the trap lifetime. Early atom interferometry experiments using atoms confined in magnetic waveguides showed that the external state coherence of the atoms decayed quite quickly, limiting interferometric measurements to times $<10 \mathrm{~ms}[6,7]$. More recent experiments using Bose condensates [8] have shown that the external state coherence can be preserved for approximately $200 \mathrm{~ms}$, where the decoherence is dominated by atom-atom interactions. Interferometry experiments using either condensed atoms in a weak trap or noncondensate atoms in a waveguide with precise angular alignments have been shown to have phase-stable interrogation times of $\approx 50 \mathrm{~ms}$, where the dephasing is induced by inhomogeneities in the confining potential [9-12].

This work demonstrates a new atom interferometer configuration that measures the differential phase shift of spatially displaced wave-packet pairs. We demonstrate phase-stable interferometry operations with up to $1 \mathrm{~s}$ interrogation time by applying the technique to atoms in a straight magnetic guide. We show that the matter-wave dephasing rate scales linearly with the wave-packet displacement, suggesting that dephasing in our interferometer is primarily caused by a weak longitudinal confinement of the atoms. We also demonstrate that the phase readout of the interferometer is less sensitive to

\footnotetext{
*Present address: Department of Physics, Massachusetts Institute of Technology, Cambridge, MA, 02139.

${ }^{\dagger}$ Present address: National Institute of Standards and Technology, Gaithersburg, MD, 20899.
}

vibration than conventional interferometery schemes, which should enable precision measurements even in noisy environments such as moving platforms.

\section{THE FOUR-PULSE GRATING ECHO SCHEME}

Typical Talbot-Lau matter-wave interferometery [13-16] employs a three-grating diffraction scheme. In the most common time-domain setup, an atomic wave packet is diffracted by a periodic potential, applied briefly at time $t=0$, into a collection of wave packets that depart from each other at multiples of the velocity $\mathbf{v}_{\mathbf{Q}}=\hbar \mathbf{Q} / m$, where $\mathbf{Q}$ is the potential's wave vector and $m$ is the atomic mass. The potential is pulsed on again at $t=T / 2$, and the different velocity classes created by the first pulse, which have now moved away from each other, are each again diffracted into multiple orders. After the second pulse, there will be pairs of wave packets whose relative velocity has been reversed from before; these will move toward each other, then overlap and interfere near time $t=T$. Those with relative velocity $n \mathbf{v}_{\mathbf{Q}}$ will generate density fringes with wave vector $\pm n \mathbf{Q}$.

As in earlier experiments [14,17], we use an off-resonant optical standing wave (SW) to create the pulsed periodic potentials and observe the resulting atomic density fringes by measuring the Bragg scattering of an optical probe. In our experiment we observe the lowest-order fringes, $n= \pm 1$, where the Bragg condition corresponds approximately to backscattering of one of the beams that forms the standing wave.

The interferometric technique presented here employs a four-pulse scheme (Fig. 1), where the additional pulse is used to halt the relative motion of the interfering wave-packet pairs. After the first pulse is applied, the situation is identical to that in the three-pulse case, with the original wave packet split into different diffraction orders corresponding to velocities $\pm n \mathbf{v}_{\mathbf{Q}}$. Here we quickly apply a second pulse after a short time $t=T_{s}$. The pairs of wave packets that we are interested inthose that will eventually interfere-are those that now have zero momentum difference; these pairs have the same velocity but are displaced in space, having moved apart by a distance $\mathbf{d}=\mathbf{v}_{\mathbf{Q}} T_{S}$ during the interval between pulses. After waiting for a time $T-2 T_{S}$, the coherence between the wave packets is measured by allowing them to interfere; a third pulse diffracts the wave packets at $t=T-T_{s}$ and the resulting interference fringe is probed around $t=T$. This four-pulse sequence can be imagined as a three-pulse sequence of length $2 T_{s}$ that is 


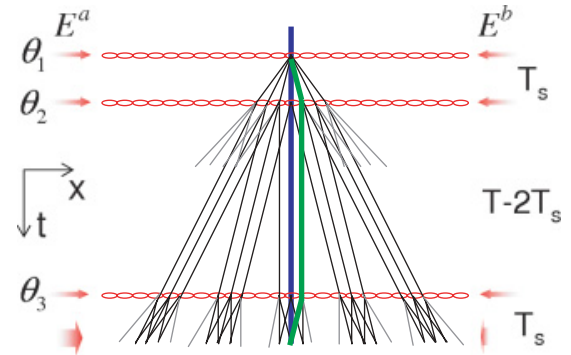

FIG. 1. (Color online) Recoil diagram of the four-pulse scheme. The light-shift potential of the standing wave is represented by the array of circles. Straight lines show the centers of the diffracted wave packets. A representative pair of interfering paths is marked with thick lines. The matter-wave interference at around $t=T$ is probed with $E^{a}$. The backscattered light [e.g., the "grating echo" $\left.E^{b}(\tau)\right]$ is mixed with a weak local field from the $E^{b}$ mode for phase retrieval.

"paused" between times $T=T_{S}$ and $T-T_{S}$, though during this time the relative phase of each separated wave-packet pair continues to be sensitive to external fields.

The existing theory of Talbot-Lau interferometry can be straightforwardly extended to this scheme. We consider the SW field formed by the traveling light fields $E^{a}$ and $E^{b}$ with associated $k$-vectors $\mathbf{k}^{a}$ and $\mathbf{k}^{b}$, with $\mathbf{Q}=\mathbf{k}^{a}-\mathbf{k}^{b}=Q \mathbf{e}_{\mathbf{x}}$. Experimentally, we measure the backscattering of light from $\mathbf{k}^{a}$ into $\mathbf{k}^{b}$; this is characterized by an electric field component $E^{b}(\tau)$ that can be expressed in terms of the atomic density operator:

$$
E^{b}(\tau)=-i g E_{p} \operatorname{Tr}\left[\hat{\rho}(T) e^{i Q\left(\hat{x}+\frac{\hat{p} \tau}{m}\right)}\right] .
$$

Here $E_{p}$ gives the amplitude of probe light from the $E^{a}$ mode; $g$ is a constant that depends on the atomic polarizability and the number of atoms participating in the interaction; $\hat{x}$ and $\hat{p}$ are the position and momentum operators, respectively, for atomic motion along $\mathbf{e}_{\mathbf{x}}$; and $\hat{\rho}(T)$ is the single-atom density matrix at time $T$. Consider an atomic sample with a rms velocity $u$ and a thermal de Broglie wavelength $l_{c}=\hbar /(m u)$. In Eq. (1) we use $\tau \sim l_{c} / v_{Q}$ to specify a coherence-length-dependent time window $\left(T-l_{c} / v_{Q}, T+l_{c} / v_{Q}\right)$, during which the atomic wave packets overlap so that the interference fringe contrast is nonzero. Experimentally, the amplitude of $E^{b}(\tau)$ is averaged during this time window to extract the magnitude of the interference fringe, which is referred to as the amplitude of the "grating echo," or $E_{g}\left(T_{s}, T\right):=\left\langle E^{b}(\tau)\right\rangle_{\tau}[18]$.

For a SW pulse in the Raman-Nath regime, where the atomic motion can be neglected for its duration, the $n$ th-order matter-wave diffraction is weighted by the amplitude $i^{n} J_{n}(\theta)$, with $J_{n}$ the $n$ th-order Bessel function and $\theta$ the time-integrated light shift or pulse area. We define $\left\{T_{1}, T_{2}, T_{3}, T_{4}\right\}=\left\{0, T_{s}, T-\right.$ $\left.T_{s}, T\right\}$ and specify the position of standing wave nodes at $T_{i}$ with the SW phase $\varphi_{i}$. The interaction of the first three SW pulses in the four-pulse interferometer can be effectively described by

$$
\hat{V}_{\mathrm{SW}}(t)=\hbar \sum_{i=1}^{3} \theta_{i} \delta\left(t-T_{i}\right) \cos \left(Q \hat{x}+\varphi_{i}\right)
$$

Since the standing-wave phases $\varphi_{i}$ involve simple algebra, we ignore them during the following discussion and reintroduce them when they become relevant.
In order to account for imperfections in the guide, we consider the one-dimensional (1D) motion of atoms along $\mathbf{e}_{\mathbf{x}}$ during the interferometry sequence to be governed by $\hat{H}=$ $\hat{H}_{0}+\hat{V}_{\mathrm{SW}}(t)$ and further $\hat{H}_{0}=\hat{p}^{2} / 2 m+V(\hat{x})$, where $V(\hat{x})$ is a general $1 \mathrm{D}$ potential. We introduce the time-dependent position and momentum operators $\hat{x}(t)=e^{i \hat{H}_{0} t} \hat{x} e^{-i \hat{H}_{0} t}$ and $\hat{p}(t)=e^{i \hat{H}_{0} t} \hat{p} e^{-i \hat{H}_{0} t}$. For $T_{s}$ to be sufficiently short, the atomic motion can be treated as free during $0<t<T-T_{s}$ and $T-T_{s}<t<T$. For a thermal atomic sample with $Q l_{c} \ll 1$, we find that, at the leading order of $Q l_{c}$, the interferometer output is related to the initial atomic density matrix $\hat{\rho}(0)$ by

$$
\begin{aligned}
E_{g}\left(T_{s}, T\right)= & -i g E_{p} J_{-1}\left(2 \theta_{3} \sin \omega_{Q} T_{s}\right) \sum_{m_{1}, m_{2}} c_{m} \operatorname{Tr} \\
& \times\left[\hat{\rho}\left(\delta x_{m}, \delta p_{m}, 0\right) e^{\frac{i}{\hbar} d \hat{p}(T)} e^{-\frac{i}{\hbar} d \hat{p}(0)}\right],
\end{aligned}
$$

where $\quad c_{m}=J_{m_{1}}\left(\theta_{1}\right) J_{m_{1}+1}\left(\theta_{1}\right) J_{m_{2}}\left(\theta_{2}\right) J_{m_{2}-1}\left(\theta_{2}\right) e^{i \phi_{m}}, \quad \phi_{m}=$ $\left(2 m_{1}+1\right) Q l_{c}+\left(2 m_{2}-1\right) \omega_{Q} T_{s}, \delta x_{m}=m_{1} v_{Q} T_{s}$, and $\delta p_{m}=$ $\left(m_{1}+m_{2}\right) Q$. In Eq. (3), $\omega_{Q}$ is the two-photon recoil frequency of atoms and we have $d=v_{Q} T_{s}$.

The second line of Eq. (3) composes a weighted sum of matter-wave correlation functions. The initial conditions of matter-wave states are specified by a density matrix $\hat{\rho}(\delta x, \delta p, 0)=e^{i(\delta x \hat{p}+\delta p \hat{x})} \hat{\rho} e^{-i(\delta x \hat{p}+\delta p \hat{x})}$ that describes an atomic ensemble that is identical to $\hat{\rho}(0)$, but with mean position and momentum shifted by $\delta x$ and $\delta p$, respectively. The correlation function gives the average overlap of wave-packet pairs propagating under an external potential displaced by $d \mathbf{e}_{\mathbf{x}}$, with one example sketched with the thick lines in Fig. 1. The correlation functions are directly analogous to the neutron-scattering correlation function discussed in Ref. [19], where momentum displacements were considered. Notice that if the uniform atomic sample has a spatial extension $L \gg \delta x$ and with thermal velocity $u \gg \delta p / m$, the original and shifted density matrix are approximately the same, and the correlation functions are approximately independent of $\delta x_{m}$ or $\delta p_{m}$. We can thus use a sum rule of Bessel functions to simplify the second line of Eq. (3):

$$
E_{g}\left(T_{s}, T\right) \approx-i \tilde{g} J_{-1}^{2}\left(2 \theta_{2} \sin \omega_{Q} T_{s}\right) \operatorname{Tr}\left[\hat{\rho}(0) e^{\frac{i}{\hbar} d \hat{p}(T)} e^{-\frac{i}{\hbar} d \hat{p}(0)}\right],
$$

where we have chosen $\theta_{2}=\theta_{3}$ and define $\tilde{g}=g E_{p} \theta_{1} Q l_{c}$.

Three features of Eqs. (3) and (4) are worth noting. First, though we have only considered the $1 \mathrm{D}$ motion of atoms in the external potential $V(x)$, the formula is readily applicable to a three-dimensional time-dependent potential $V(\mathbf{r}, t)$ as long as the external potential contributes negligibly to the differential phase shift of wave-packet pairs during $0<t<T_{s}$ and $T-T_{s}<t<T$.

Second, the reduction from Eq. (3) to Eq. (4) requires that the correlation functions be insensitive to momentum transferred by the SW pulses. This condition is very well satisfied if the displacements are much smaller than the position and momentum spreadings of the atomic gas itself since $\hat{\rho}(\delta x, \delta p, 0) \approx \hat{\rho}(0)$. In addition, the approximation is particularly well satisfied if the potential is periodic at small wavelengths [20,21].

Finally, notice that both Eq. (3) and Eq. (4) can be evaluated semiclassically by replacing $\operatorname{Tr}\left[\hat{\rho}(0) e^{\frac{i}{\hbar} d \hat{p}(T)} e^{-\frac{i}{\hbar} d \hat{p}(0)}\right]$ 
with $\left\langle e^{\frac{i m d}{h}[v(T)-v(0)]}\right\rangle_{c}$, where $v(t)$ gives the classical velocity of atoms along $\mathbf{e}_{\mathbf{x}}$ and $\langle\cdots\rangle_{c}$ gives the classical ensemble average over the atomic initial conditions.

Now consider a weak quadratic potential $V(x)=\max +$ $\frac{1}{2} m \omega_{l}^{2} x^{2}$ with an acceleration force $m a$ and with $\omega_{l} T \ll 1$ to model the potential variation along the nearly free (axial) direction of propagation in the magnetic guide, also assuming an atomic sample with a Gaussian spatial distribution along $\mathbf{e}_{\mathbf{x}}$ given by $\rho(x)=e^{-x^{2} /\left(2 L^{2}\right)}$. The expected amplitude of the grating echo signal is then found to oscillate with $T_{s}$ and to decay as a Gaussian with the total interrogation time $T$ :

$$
\begin{aligned}
E_{g}\left(T_{s}, T\right) \approx & -i \tilde{g} J_{-1}^{2}\left(2 \theta_{2} \sin \omega_{Q} T_{s}\right) \\
& \times e^{-\frac{1}{2}\left(\frac{m d}{\hbar} \omega_{l}^{2} T L\right)^{2}} e^{i\left(\frac{m d}{\hbar} a T+\varphi_{1,2}-\varphi_{3,4}\right)} .
\end{aligned}
$$

(Here we have reintroduced the standing-wave phase in Eq. (2), where $\varphi_{i, j}=\varphi_{i}-\varphi_{j}$.)

\section{EXPERIMENTAL SETUP}

The experimental apparatus is described in detail in Ref. [18]. A straight two-dimensional quadruple magnetic field with a transverse gradient of $70 \mathrm{G} / \mathrm{cm}$ is generated by four $200 \times 100 \times 1.56 \mathrm{~mm}^{3}$ permalloy foils poled in alternating directions. Approximately $10^{7}$ laser-cooled ${ }^{87} \mathrm{Rb}$ atoms in the ground-state $F=1$ hyperfine level are loaded into this magnetic guide, resulting in a cylindrically shaped atom sample $1 \mathrm{~cm}$ long and $170 \mu \mathrm{m}$ wide. The average transverse oscillation frequency of the atoms in the guide is on the order of $80 \mathrm{~Hz}$, estimated by displacement-induced oscillations of the atomic sample using absorption images. A very weak harmonic potential along the guiding direction is estimated to be $\omega_{l} \sim 2 \pi \times 0.08 \mathrm{~Hz}$ [18].

The SW fields formed by two counterpropagating laser beams with diameters of $1.6 \mathrm{~mm}$ are aligned to form a standing wave with $k$-vector along the magnetic guide direction $\mathbf{e}_{\mathbf{x}}$. Precise angular adjustment is achieved by tuning the orientation of the magnetic guide using two rotation stages to within $2 \times 10^{-4} \mathrm{rad}$. The optical fields are detuned $120 \mathrm{MHz}$ above the $F=1-F^{\prime}=2 \mathrm{D} 2$ transition. We choose the SW pulse with typical pulse area of $\theta \sim 0.8-3.0$, and with duration of $300 \mathrm{~ns}$, to be deep in the thin-lens regime of the $25-\mu \mathrm{K}$ atomic sample. With this pulse duration, the fraction of atoms contributing to the final interference fringe is typically limited by SW diffraction efficiency to about $10 \%$. We probe the $\lambda / 2$ atomic density grating at around time $t=T$ by turning on only one of the traveling wave beams; the other beam is attenuated and shifted by $6 \mathrm{MHz}$ to serve as an optical local oscillator, where the combined intensity is measured using a fiber-coupled avalanche photodetector. The beat signal is measured and numerically demodulated using the 6-MHz rf reference to recover the grating echo signal $E_{g}\left(T_{s}, T\right)=$ $C\left(T_{s}, T\right) e^{i \varphi\left(T_{s}, T\right)}$. The interferometer signal amplitude $C\left(T_{s}, T\right)$ and phase $\varphi\left(T_{s}, T\right)$ are measured for different interferometer parameters.

\section{RESULTS AND DISCUSSION}

According to Eq. (5), the prefactor $J_{1}^{2}\left(2 \theta \sin \omega_{Q} T_{s}\right)$ in the backscattering amplitude is an oscillatory function of $T_{s}$, with
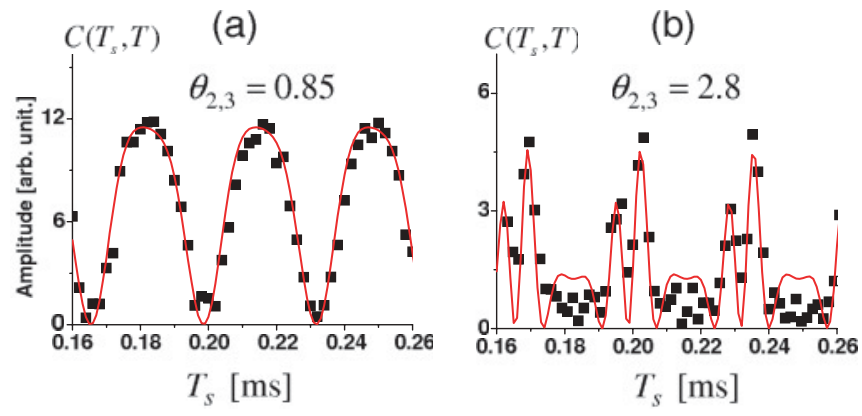

FIG. 2. (Color online) Examples of interferometry signal amplitude $C\left(T_{s}, T\right)$ oscillation vs $T_{s}$ at fixed $T$. Scatterplots are from experiments. Solid lines are calculated according to an extension of Eq. (5) with complex pulse area $\tilde{\theta}=\theta(1+0.025 i)$. (a) $\theta=0.85$ and $T=50 \mathrm{~ms}$. (b) $\theta=2.8$ and $T=30 \mathrm{~ms}$. The data were taken during different a period of experiments with different signal-to-noise ratios.

the periodicity determined by $2 \omega_{Q}=2 \pi / 33.15 \mu \mathrm{s}^{-1}$. The amplitude oscillation is reproduced experimentally; two examples are plotted in Fig. 2, where $T_{s}$ is varied from 0.16 to $0.26 \mathrm{~ms}$. In Fig. 2(a) a relatively small SW pulse area, $\theta \approx 0.85$, was chosen so that the Bessel function is approximately linear. Correspondingly, we see that the oscillation is approximately sinusoidal. In Fig. 2(b) a strong SW pulse with area $\theta \approx 2.8$ was chosen and the Bessel function becomes highly nonlinear. Nevertheless, the experimental data still fit the theoretical expectation from Eq. (5) fairly well. The values for the pulse area in the calculation were found to be in agreement with the SW pulse intensity-duration products. The solid lines in Fig. 2 were calculated according to an extension of Eq. (5) with a complex SW pulse area including an imaginary part to account for the optical pumping effect at the $120-\mathrm{MHz}$ SW detuning [18].

With fixed $T_{s}$ at the peak values of the amplitude oscillations, we now consider the dependence of the interferometer signals on the total interrogation time $T$. Figure 3 gives examples of the interferometer amplitude decay $C(T)$ and phase shift $\varphi(T)$ at various $d=v_{Q} T_{s}$. From Fig. 3(a) we see that the amplitude decay is slower for smaller $d$, while all the $C(T)$ fit fairly well to Gaussian decay, in agreement with Eq. (5) derived from a weak harmonic confinement model. In Fig. 3(b) we see that the phase readout is a linear function
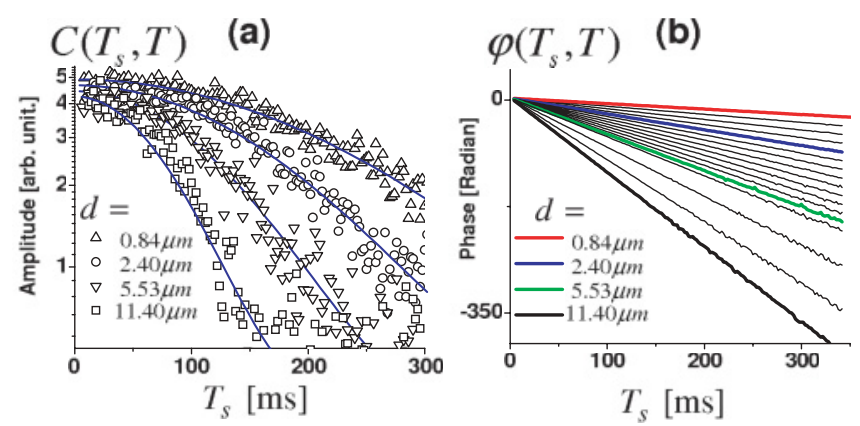

FIG. 3. (Color online) (a) Backscattering amplitude decay. The scatterplots give $C(T)$ at four different displacements $d$. The solid lines are due to Gaussian fit. (b) Interferometry phase shift $\varphi(T)$ vs $T$ at different displacements $d$. Four of the data traces from the same experiments as those in panel (a) are plotted with thick lines. 

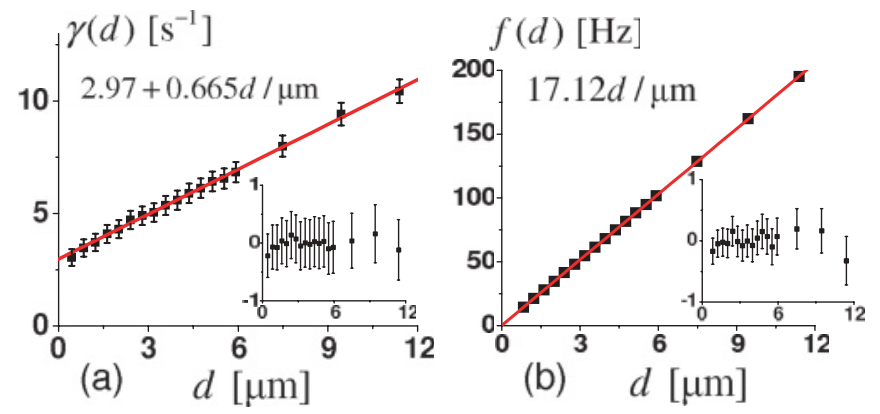

FIG. 4. (Color online) (a) Backscattering amplitude decay rate $\gamma(d)$ [extracted from a Gaussian fit of $C(T)$ data such as those from Fig. 3(a)] vs wave-packet displacement $d$. (b) Interferometry phase shift rate $f(d)=\frac{d \varphi(T)}{d T}$ [extracted from a linear fit of $\varphi(T)$ data from Fig. 3(b)] vs $d$. The insets give the residuals of the linear fits.

of interrogation time $T$, which is also in agreement with Eq. (5). By applying Eq. (5) to the observed phase shifts, we consistently retrieve an acceleration $a=83.4 \mathrm{~mm} / \mathrm{s}^{2}$ for different $d$. The acceleration is due to a small component of gravity along the SW and magnetic guide direction $\mathbf{e}_{\mathbf{x}}$ [18], as confirmed by varying the tilt angle of the apparatus.

We extract the amplitude decay rate $\gamma(d)$ by fitting the $C(T)$ decay data with $C(T) \propto e^{-[\gamma(d) T]^{2}}$. The dephasing rate $\gamma(d)$ is plotted versus the displacement $d$ in Fig. 4(a). The $d$-dependence of $\gamma(d)$ shows good agreement with a linear fit. According to Eq. (5), for weak confinement along $\mathbf{e}_{\mathbf{x}}$ with $\omega_{l} \sim 2 \pi \times 0.08 \mathrm{~Hz}$ and for $L \sim 2.5 \mathrm{~mm}$ of our $1-\mathrm{cm}$ atomic sample, we expect $\gamma(d) \sim m \omega_{l}^{2} d L /(\hbar \sqrt{2}) \sim$ $0.6 d / \mu \mathrm{m} \mathrm{s}^{-1}$. This agrees with the experimentally measured $\gamma(d)=(2.97+0.665 d / \mu \mathrm{m}) \mathrm{s}^{-1}$ according to Fig. 4(a). The offset of $\gamma(d \rightarrow 0)=2.97 \mathrm{~s}^{-1}$ is partly due to the escape of atoms from the interaction zone via collisions with the walls of the 4-cm vacuum glass cell, which, if fit to a Gaussian, would give $\tilde{\gamma}(d \rightarrow 0) \sim 1.6 \mathrm{~s}^{-1}$. The remaining discrepancy is likely due to the inaccuracy of the Gaussian fit, which is based on the assumption of a weak harmonic perturbation $V(x)$ in Eq. (5). For small $d$ and thus a small dephasing rate, local anharmonicity in $V(x)$ might become important. Indeed, for long interaction time $T$, the decay exhibits an exponential feature, which is clearly seen in Fig. 5(a), where the amplitude decay $C(T)$ with $d=0.418 \mu \mathrm{m}$ and $T_{s}=35.4 \mu$ s is plotted. For such a small wave-packet displacement $d$, the phase of the
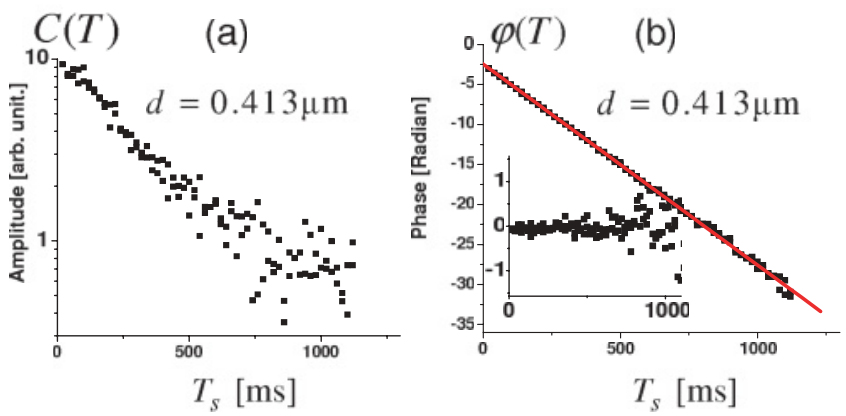

FIG. 5. (Color online) Interferometry readouts $C(T)$ and $\varphi(T)$ at $d=0.413 \mu \mathrm{m}$. (a) $C(T)$ vs $T$; (b) $\varphi(T)$ vs $T$, with inset giving the residual after a linear fit.
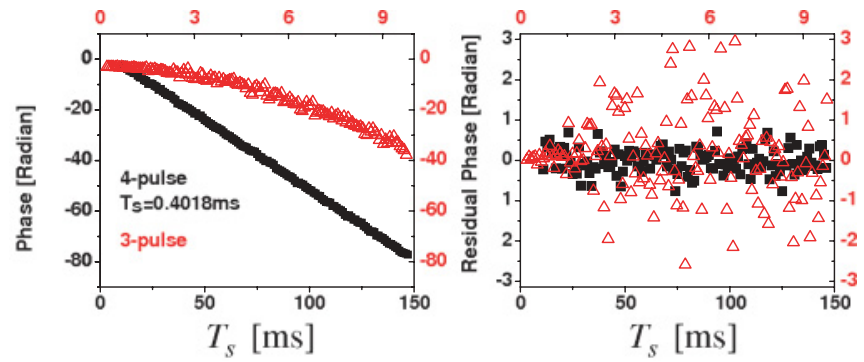

FIG. 6. (Color online) Interferometry phase readouts $\varphi(T)$ for a three-pulse (open triangles, right and top axes) and a four-pulse (solid squares, left and bottom axes; $T_{s}=0.4018 \mathrm{~ms}$ ) configurations. Panel (a) shows the phase readouts, and panel (b) gives the residual of a quadratic (three-pulse) and a linear (four-pulse) fit.

backscattering signal remains stable for $T>1 \mathrm{~s}$, as shown in Fig. 5(b).

Last, we consider the effect of phase noise in the SW on the sensitivity of our device, induced, for example, by vibrations of mirrors in the $\mathrm{SW}$ path. For $T \gg T_{s}$, the $\mathrm{SW}$ phase variation due to time-dependent changes in mirror positions is given by $\varphi_{1,2}$, which is not correlated with $\varphi_{3,4}$. If we specify the SW phase at time $t$ with $\phi(t)$ such that $\varphi_{i}=\phi\left(T_{i}\right)$, the mirror-vibration-induced interferometer phase noise is given by $N_{\varphi, \text { mirror }}\left(T_{s}, T\right)=\sqrt{2\left\langle\left[\phi(t)-\phi\left(t+T_{s}\right)\right]^{2}\right\rangle_{t}}$, which does not depend on $T$. This is different from a three-pulse atom interferometer with mirror-induced phase noise given by $N_{\varphi \text {,mirror }}^{\prime}(T)=\sqrt{\left\langle[\phi(t)-2 \phi(t+T / 2)+\phi(t+T)]^{2}\right\rangle_{t}}$, where increases in sensitivity due to increases in interaction time necessarily also result in increases in phase noise. In contrast, in the four-pulse scheme considered here, $T$ can be increased to improve the sensitivity while keeping $N_{\varphi \text {, mirror }}\left(T_{S}\right)$ unaffected.

In Fig. 6, we compare the three- and four-pulse interferometer phase readouts under the same noisy environmental conditions. A white-noise voltage source is filtered to eliminate frequencies below $100 \mathrm{~Hz}$, then amplified and applied to a piezodriven mirror in the SW optical path. As shown in Fig. 6, the mirror vibration randomizes the phase of the three-pulse interferometer for $T>5 \mathrm{~ms}$. Under the same conditions, the phase of the four-pulse interferometer is stable for times longer than $150 \mathrm{~ms}$. In this case the acceleration sensitivity of the four-pulse interferometer $\delta \varphi / \delta a \sim 1 \mathrm{rad} /\left(\mathrm{mm} / \mathrm{s}^{2}\right)$ at $T=150 \mathrm{~ms}$ exceeds that for the three-pulse case of $\delta \varphi / \delta a \sim$ $0.4 \mathrm{rad} /\left(\mathrm{mm} / \mathrm{s}^{2}\right)$ at $T=10 \mathrm{~ms}$. The insensitivity of the four-pulse scheme to low-frequency mirror vibrations is a feature of speedometers, as shown in Eqs. (4) and (5) in the semiclassical limit with the phase proportional to the velocity during the interrogation time $T$.

\section{SUMMARY}

We have demonstrated a four-pulse grating echo interferometer scheme to study the dephasing effects for atoms confined in a magnetic guide. We find a linearly reduced dephasing rate at reduced wave-packet displacements, indicating that the matter-wave dephasing is due to very weak potential variation along the waveguide in our setup. We have demonstrated phase stability for an interferometry sequence with total 
interrogation time exceeding $1 \mathrm{~s}$. We also showed that a four-pulse interferometer can provide acceleration measurements with very long integration times that are insensitive to apparatus vibrations, though it is important to note that the sensitivity of the interferometer scheme we describe is compromised by the small wave-packet separations [10].

In the future, such a system could study the quantum stability of wave packets due to displaced potentials [19] by deliberately introducing time-dependent variations in the potential along the waveguide direction [20,21]. Instead of measuring the mixed-state correlation functions, a fidelity-type measurement [19] can be performed with sub-recoil cooled atoms occupying a single matter-wave state, where velocityselective beam-splitting schemes can be applied [22,23].

\section{ACKNOWLEDGMENTS}

We thank Professor Eric Heller and Dr. Cyril Petitjean for helpful discussions. This work is supported in part by MURI and the Defense Advanced Research Projects Agency from the Department of Defense, ONR, and the US Department of the Army, Agreement No. W911NF-04-1-0032, by NSF, and by the Charles Stark Draper Laboratory.
[1] Atom Interferometry, edited by P. R. Berman (Academic, Cambridge, 1997).

[2] A. Peters, K. Y. Chung, and S. Chu, Metrologia 38, 25 (2001).

[3] J. F. Clauser, Physica B 151, 262 (1988).

[4] D. Muller, D. Anderson, R. Grow, P. D. D. Schwindt, and E. Cornell, Phys. Rev. Lett. 83, 5194 (1999).

[5] N. H. Dekker, C. S. Lee, V. Lorent, J. H. Thywissen, S. P. Smith, M. Drndić, R. M. Westervelt, and M. Prentiss, Phys. Rev. Lett. 84, 1124 (1999).

[6] Y.-J. Wang, D. Z. Anderson, V. M. Bright, E. A. Cornell, Q. Diot, T. Kishimoto, M. G. Prentiss, R. A. Saravanan, S. R. Segal, and S. Wu, Phys. Rev. Lett. 94, 090405 (2005).

[7] S. Wu, E. J. Su, and M. G. Prentiss, Eur. Phys. J. D 35, 111 (2005).

[8] G.-B. Jo, Y. Shin, S. Will, T. A. Pasquini, M. Saba, W. Ketterle, D. E. Pritchard, M. Vengalattore, and M. Prentiss, Phys. Rev. Lett. 98, 030407 (2007).

[9] O. Garcia, B. Deissler, K. J. Hughes, J. M. Reeves, and C. A. Sackett, Phys. Rev. A 74, 031601(R) (2006).

[10] J. H. T. Burke, B. Deissler, K. J. Hughes, and C. A. Sackett, Phys. Rev. A 78, 023619 (2008).

[11] S. Wu, E. J. Su, and M. G. Prentiss, Phys. Rev. Lett. 99, 173201 (2007).
[12] J. H. T. Burke and C. A. Sackett, Phys. Rev. A 80, 061603(R) (2009).

[13] J. F. Clauser and M. W. Reinsch, Appl. Phys. B 54, 380 (1992).

[14] S. B. Cahn, A. Kumarakrishnan, U. Shim, T. Sleator, P. R. Berman, and B. Dubetsky, Phys. Rev. Lett. 79, 784 (1997).

[15] D. V. Strekalov, A. Turlapov, A. Kumarakrishnan, and T. Sleator, Phys. Rev. A 66, 023601 (2002).

[16] B. Brezger, L. Hackermüller, S. Uttenthaler, J. Petschinka, M. Arndt, and A. Zeilinger, Phys. Rev. Lett. 88, 100404 (2002).

[17] T. W. Mossberg, R. Kachru, E. Whittaker, and S. R. Hartmann, Phys. Rev. Lett. 43, 851 (1979).

[18] S. Wu, Ph.D. thesis, Harvard University, 2007.

[19] C. Petitjean, D. V. Bevilaqua, E. J. Heller, and P. Jacquod, Phys. Rev. Lett. 98, 164101 (2007).

[20] F. L. Moore, J. C. Robinson, C. F. Bharucha, B. Sundaram, and M. G. Raizen, Phys. Rev. Lett. 75, 4598 (1995).

[21] S. Wu, A. T. Tonyushkin, and M. G. Prentiss, Phys. Rev. Lett. 103, 034101 (2009).

[22] S. Wu, Y.-J. Wang, Q. Diot, and M. Prentiss, Phys. Rev. A 71, 043602 (2005).

[23] D. M. Giltner, R. W. McGowan, and S. A. Lee, Phys. Rev. Lett. 75, 2638 (1995). 